\title{
Celestial frame instability in VLBI analysis and its impact on geophysics
}

\author{
S. B. Lambert ${ }^{1}$, V. Dehant ${ }^{2}$, and A.-M. Gontier ${ }^{1}$ \\ 1 Paris Observatory, Systèmes de Référence Temps Espace (SYRTE), CNRS/UMR8630, 75014 Paris, France \\ e-mail: [sebastien.lambert; anne-marie.gontier]@obspm.fr \\ 2 Royal Observatory of Belgium, 1180 Brussels, Belgium \\ e-mail: v.dehant@oma.be
}

Received 16 August 2007 / Accepted 7 January 2008

\section{ABSTRACT}

\begin{abstract}
Context. Derivation of Earth's orientation parameters through VLBI analysis needs to handle the positions of the radio sources and the celestial reference frame correctly, so that radio source positional instabilities will not unduly perturb the nutation estimates or all the subsequent geophysical analysis.

Aims. This work aims at estimating the magnitude of these perturbations. It also yields new determinations of the resonant frequencies associated with the Earth's fluid outer and inner cores.

Methods. We generate several VLBI global solutions, analyzing VLBI delays accumulated from 1984 to 2007, and using different constraints on the radio source positions, to obtain nutation offset time series. Then we analyze the nutation time series obtained from each global solution, from which we extract some prominent spectral components reflecting the non rigid Earth's response to the external tidal potential. Finally, we deduce values of the outer and inner cores' resonant frequencies from each solution.

Results. We show that the error propagated in VLBI analysis that is due to instability in the celestial reference frame can produce an additional error in the estimates of nutation spectral components of $15 \mu$ as for the 18.6-yr term, and decreasing for shorter periods. This leads to an uncertainty of a few tenth of a day on the RFCN period and of less than 200 on its quality factor, and to an uncertainty of roughly 100 days on the FICN period and of 100 on its quality factor. The values yielded for the outer and inner cores' resonant frequencies are nevertheless close to the MHB values within the error bars.
\end{abstract}

Key words. reference systems - Earth

\section{Introduction}

Very long baseline radio interferometry (VLBI) is currently the only geodetic technique giving direct access to the direction of the Earth's figure axis with respect to a space-fixed frame of reference. The time variations of this axis, also known as the precession-nutation, mainly reflect the response of the non rigid Earth to the gravitational forcing of the celestial bodies (Moon, Sun, planets). The complex descriptions of the Earth's structure associated with fitting of some geophysical parameters to VLBI data allow researchers to build up powerful precession-nutation models. The most accurate one, developed by Mathews et al. (2002), was adopted as the reference model by the International Astronomical Union (IAU) in 2000 and is also referred to as MHB.

The VLBI-derived nutation time series against MHB leave residuals of $\sim 200$ micro arcseconds ( $\mu$ as) in rms. Exploring these differences reveals imperfections of both the theory and of the VLBI observing and analysis strategies. Some phenomena appear to be unpredictable, like the free motion associated with the free rotation of the fluid outer core inside the mantle (retrograde free core nutation, or RFCN) excited by external geophysical fluids (see, e.g., Gegout et al. 1998; Herring et al. 2002; Vondrák et al. 2005; Lambert 2006). Likewise, some tidal terms not completely described by the theory could show up. A thorough study of these terms would obviously bring up new information about the non rigidity of the Earth. Recent works from
Vondrák et al. (2005) and Lambert \& Dehant (2007) have investigated the RFCN resonant frequency, taking advantage of VLBI data sets longer than those used in MHB by $\sim 5$ years. Extending such works to the free inner core nutation (FICN) is the first goal of this paper.

However, at this level of accuracy, errors from VLBI analysis have to be considered. These errors come from the models used in the data reduction, as well as from the treatment of local and global parameters and from the realization of global reference frames. Indeed, VLBI orients the terrestrial coordinate system with respect to the celestial coordinate system, the latter being materialized by radio source positions. One can obviously consider that a reference catalogue, e.g., the conventional IAUadopted International Celestial Reference Frame (ICRF) of Ma et al. (1998) or its latest extension ICRF Ext.2 (Fey et al. 2004), gives the right positions of all the radio sources. In such a way, the analysts completely evade evolutions of radio source structure (e.g., plasma jets that can shift the radio center by tens of $\mu$ as) and, more generally, what will be referred to as positional instabilities in the following. Multi-session VLBI analysis offers the possibility estimating both Earth orientation parameters and radio source positions. Nevertheless, several ways exist to do these estimations, and each can perturb the output nutation time series differently, and therefore any extracted geophysical result. Estimating the magnitude of these perturbations, as well as their effects on the estimates of the outer and inner cores' resonant frequencies, is the second goal of this paper. 
Table 1. Characteristics of the VLBI solutions used in this work. MFV: 247 stable sources of Feissel-Vernier et al. (2006); ICRF: 212 ICRF defining sources of Ma et al. (1998).

\begin{tabular}{|c|c|c|c|c|c|c|c|c|c|c|c|c|c|c|c|}
\hline & \multicolumn{3}{|c|}{ No. sources } & \multirow[t]{2}{*}{$\overline{\mathrm{NNR}}$} & \multirow{2}{*}{$\begin{array}{r}\text { Postfit rms } \\
\text { ps } \\
\end{array}$} & \multirow{2}{*}{$\begin{array}{r}\operatorname{rms~} \delta X \\
\mu \text { as }\end{array}$} & \multirow{2}{*}{$\begin{array}{r}\mathrm{rms} \delta Y \\
\mu \mathrm{as}\end{array}$} & \multicolumn{2}{|c|}{$\overline{\overline{A_{1}}}$} & \multicolumn{2}{|c|}{$\overline{A_{2}}$} & \multicolumn{2}{|c|}{$\overline{A_{3}}$} & \multicolumn{2}{|c|}{$\overline{\mathrm{d} z}$} \\
\hline & fixed & global & local & & & & & $\mu$ as & \pm & $\mu$ as & \pm & $\mu$ as & \pm & $\mu$ as & \pm \\
\hline $\bar{A}$ & 816 & 0 & 0 & - & 24.0 & 165 & 167 & - & - & - & - & - & - & - & - \\
\hline B & 0 & 816 & 0 & ICRF & 23.6 & 166 & 173 & -22 & 14 & 55 & 15 & 9 & 11 & 17 & 13 \\
\hline $\mathrm{C}$ & 0 & 816 & 0 & MFV & 23.6 & 161 & 169 & 40 & 9 & 47 & 8 & -5 & 6 & -8 & 8 \\
\hline D & 0 & 521 & 295 & ICRF & 23.6 & 162 & 170 & 29 & 9 & 46 & 9 & 3 & 8 & 0 & 9 \\
\hline $\mathrm{E}$ & 0 & 521 & 295 & MFV & 23.6 & 161 & 169 & 33 & 8 & 38 & 7 & -9 & 6 & -11 & 7 \\
\hline $\mathrm{F}$ & 0 & 653 & 163 & ICRF & 23.2 & 167 & 168 & 20 & 9 & 68 & 11 & 7 & 8 & -8 & 9 \\
\hline G & 0 & 653 & 163 & MFV & 23.2 & 166 & 168 & 19 & 8 & 57 & 9 & 3 & 6 & -14 & 8 \\
\hline
\end{tabular}

Our work first presents the analysis of VLBI delays since 1984 through several, solutions each using different analysis strategies for handling radio source positions (Sect. 2). Then we fit a number of forced nutation terms and deduce the RFCN and FICN resonant frequencies, together with their respective sensitivity to the analysis strategies (Sect. 3).

\section{Processing of VLBI delays}

Our solutions all have the same technical description except for the constraints applied to the radio source coordinates. Sessions span 1984.01-2007.45 (2995 sessions, totaling 3.4 million delays). The elevation cut-off is $6^{\circ}$. No-net rotation and no-net translation constraints are applied to the positions and velocities of 26 stations with a priori positions and velocities taken from the VTRF 2005 catalogue (Nothnagel et al. 2006). The motion of the Fairbanks station (which participates in almost 1200 sessions) has been considered as non linear due to the coseismic displacement after the 2002 Denali fault earthquake that gave discontinuities both in position and velocity (MacMillan \& Cohen 2004). A priori zenith delay is given by the Niell (1996) mapping functions. A priori precession and nutation comply with the IAU 2000 resolutions (the nutation model is the one of Mathews et al. 2002) and the NRO-based coordinate transformation between terrestrial and celestial coordinate systems is implemented (Capitaine et al. 2003). While we estimate mean station coordinates and velocities over the entire time span (station coordinates and velocities are thus called "global parameters"), polar motion coordinates, UT1, length of day and nutation offsets are all estimated once per session ("local parameters"). Radio source positions are discussed further. Zenith delay and gradients are estimated every $20 \mathrm{~min}$ and $6 \mathrm{~h}$, respectively. All the calculations use the Calc 10.0/Solve 2006.06.08 geodetic VLBI analysis software package and are led at the Paris Observatory IVS Analysis Center, part of the International VLBI Service for Geodesy and Astrometry (IVS, Schlueter et al. 2002).

The radio source positions need to be constrained to at least ensure that the celestial reference frame is non rotating with respect to the far universe (realized by applying a no-net rotation, or NNR, constraint to a set of radio source coordinates), and its axes are stable in time. For this purpose, Ma et al. (1998) provided a subset of 212 ICRF "defining" sources, on the basis of VLBI observations until 1995. Gontier et al. (2002), and Feissel-Vernier (2003), Feissel-Vernier et al. (2006, also referred to as MFV in the following) have all sought a better subset, with the result some of the 212 ICRF defining sources are shown to be unstable (strong time variability of the astrometric position in $X$-band). In the MFV work, the authors elected 247 sources whose coordinate time series can be considered as stable over the observational time span. A celestial reference frame realized through these stable sources is therefore expected to be more stable in time than the current ICRF.

The strategies concerning the estimation of radio source coordinates and the choice of the subset on which the NNR is applied are explained in Table 1. In a first solution (referred to as solution A), all sources are fixed to their ICRF Ext. 2 positions. In another two solutions, coordinates of all sources are estimated as global parameters and the NNR is alternatively done on the 212 ICRF defining sources (solution B) and on the 247 MFV stable sources (solution C). Solutions B and C therefore differ only in the subset of sources to which the NNR is applied. The coordinates of these sources, although constrained by the NNR, are estimated as global parameters. The same remark can be made about solutions $\mathrm{D}$ and $\mathrm{E}$, and to $\mathrm{F}$ and $\mathrm{G}$ as described in the next paragraph.

Another two solutions (D and E) consider that some poorly observed sources having less than 20 observations in less than 2 sessions could get unreliable global estimates of their positions. We therefore estimate one position per session for these sources. Nevertheless, all these solutions do not really treat the radio source positional instabilities, so we process two solutions $\mathrm{F}$ and $\mathrm{G}$, wherein the coordinates of 163 sources elected as highly unstable by Feissel-Vernier et al. are downgraded as local parameters. For all solutions, the a priori catalogue is the ICRF Ext.2 where the coordinates of the 212 ICRF defining sources have been kept identical to those in the ICRF. In addition to the NNR constraint, the source coordinates are individually constrained to stay within small circles of $\sim 2$ milliarcsec (mas) diameter. We have chosen this value after several tests. Although solutions $\mathrm{C}$ to $\mathrm{G}$ are not sensitive to this constraint (i.e., the NNR is sufficient to keep the celestial frame consistent and avoid sources going very far from their initial position, which would be unrealistic), solution B needs such a very tight value that without it a global rotation of the frame reaching $\sim 0.6$ mas shows up. That the solution $\mathrm{C}$ is not sensitive to this constraint shows that the 247 MFV stable sources define a better reference frame than the 212 ICRF defining sources, at least for analizing the current VLBI observational material.

Figure 1 shows the observational history of the radio sources used in this analysis. One must mention here that the set of unstable sources overlaps the 212 ICRF defining sources: 60 unstable sources are defining sources in the ICRF. One can see in particular that the unstable sources represent a significant part of the observations, namely 695361 delays (21\% of the total), whereas the 295 poorly observed sources only total 2014 delays (far less than $1 \%$ ). One can also compare the corresponding number of delays for the sources whose coordinates are estimated as global parameters: the 521 "global" sources of E represent 3348127 observations, whereas the 653 sources of G represent 2654780 observations. 
Table 2. Wrms of the difference between solutions $i$ and $j$.

\begin{tabular}{lrrrrrr}
\hline \hline & $\mathrm{A}$ & $\mathrm{B}$ & $\mathrm{C}$ & $\mathrm{D}$ & $\mathrm{E}$ & $\mathrm{F}$ \\
\hline $\mathrm{B}$ & $0.030 / 0.022$ & & & & & \\
$\mathrm{C}$ & $0.028 / 0.021$ & $0.000 / 0.000$ & & & & \\
$\mathrm{D}$ & $0.028 / 0.021$ & $0.001 / 0.001$ & $0.001 / 0.001$ & & & \\
$\mathrm{E}$ & $0.028 / 0.021$ & $0.001 / 0.001$ & $0.001 / 0.001$ & $0.000 / 0.000$ & & \\
$\mathrm{~F}$ & $0.038 / 0.030$ & $0.029 / 0.027$ & $0.026 / 0.024$ & $0.026 / 0.025$ & $0.026 / 0.024$ & \\
$\mathrm{G}$ & $0.037 / 0.030$ & $0.028 / 0.027$ & $0.025 / 0.024$ & $0.025 / 0.024$ & $0.025 / 0.024$ & $0.000 / 0.000$ \\
\hline
\end{tabular}

First value is for $\Delta X$, second value is for $\Delta Y$. Unit: mas.
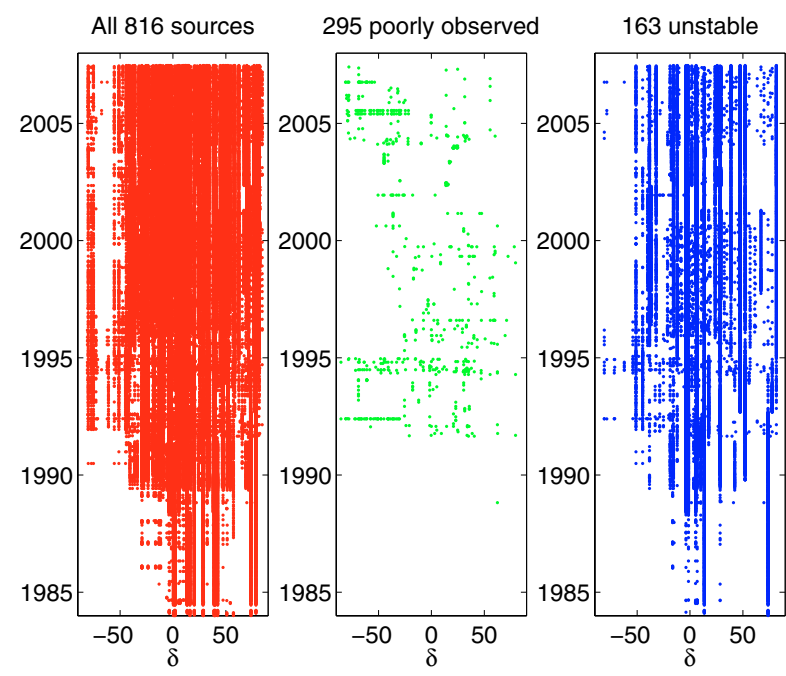

Fig. 1. Observational history of (left) all the 816 sources, (middle) only the 295 sources having less than 20 observations in less than 2 sessions, and (right) the 163 unstable sources of Feissel-Vernier et al. (2006).

Table 1 also reports on statistics concerning the postfit rms delay of each solution (Col. 6). All postfit rms delays are around 23 ps with small non statiscally significant variations. Columns 7 and 8 provide the rms of the nutation offset time series $\delta X$ and $\delta Y$, which are offsets to the MHB model on the $X$ and $Y$ components, respectively. The smallest rms for $\delta X$ are on solutions $\mathrm{C}$ and $\mathrm{E}$ (both using the stable MFV sources in the NNR). This is, however, no longer true for $\delta Y$, but differences are only a few $\mu$ as. The last eight columns of Table 1 give the parameters of the transformation between the a priori and the estimated radio source catalogues. This transformation is modeled by a sequence of three rotations around the $X, Y$, and $Z$-axes of the ICRF (described by the angles $A_{1}, A_{2}$, and $A_{3}$, respectively) and completed by the adjustment of drifts in right ascension and declination, both functions of the declination and a bias in declination $(\mathrm{d} z)$ intending to absorb some systematic error in declination (due to, e.g., troposphere delay mismodeling and network deficiencies). (This algorithm of comparison is the one currently in use at the ICRS Product Center of the IERS, see, e.g., IERS 1996. Although the complete transformation includes two slopes in right ascension and in declination, these slopes appear to be negligible so we have chosen not to estimate them.) One hundred two sources that are common to the ICRF 212 defining sources and to the MFV set have been used for the transformation. While $A_{3}$ and $\mathrm{d} z$ are not significant, $A_{1}$ and $A_{2}$ appear to have significant values when one considers the associated formal errors. One observes that the solutions having a NNR with respect to the MFV sources show lower $A_{2}$ angles. This is, however, not the case for $A_{1}$ : solutions $\mathrm{D}$ to $\mathrm{G}$ give almost the same value for this angle, but solution $\mathrm{C}$ gives a $A_{1}$ significantly higher than $\mathrm{B}$. Note that solution A obviously shows no rotation with respect to the ICRF Ext.2 since no source coordinates are estimated. However, the source coordinates are somewhat falsified by this procedure since additional observations coming after the release of the ICRF Ext. 2 permit different (and better) estimates of their position. One can therefore expect that the nutation estimates given by solution A will also be perturbed.

\section{Effect on nutation and on the estimates of the outer and inner cores resonant frequencies}

This section investigates the various nutation time series $A$ to $G$ obtained from the different VLBI analyses explained previously. Its aim is twofold: determining the influence of the analysis strategy on the nutation amplitude estimates, and propagating this influence onto the outer and inner cores' resonant frequencies.

Table 2 displays the wrms of the differences $\Delta X_{i j}=\delta X_{i}-\delta X_{j}$ and $\Delta Y_{i j}=\delta Y_{i}-\delta Y_{j}$ for solutions $i$ and $j$. Figure 2 illustrates these differences for some solutions along with the differences between their respective formal errors. The patterns can reach up to 0.2 mas in the early years. Essentially, noise and slow variations show up and will affect only the longer nutation periods. The difference between A and other solutions reflects the introduction of correlations caused by the joint estimates of source coordinates (either global or local) and Earth orientation parameters.

It can appear as paradoxical that $\mathrm{C}$ minus $\mathrm{E}$ and $\mathrm{C}$ minus $\mathrm{G}$ look so different. Nevertheless, we have already pointed out that the 163 unstable sources constitute a drastically different subset from the 295 poorly observed sources in terms of observational history (see Fig. 1). The number of local parameters estimated from solution $\mathrm{E}$ is $649 \times 2$, as the 295 poorly observed sources appears only in about 250 sessions and generally more than one source of this subset is observed in a given session. For the 163 unstable sources of solution $\mathrm{G}$, the number of sessions is about 2812 , which yields $26358 \times 2$ local parameters. It turns out that solution $\mathrm{E}$ only estimates a moderate number of local parameters compared to solution G. Higher correlations between parameters are therefore expected in the latter solution. This could partly explain the observed differences showing up between the plots of $\mathrm{C}$ minus $\mathrm{E}$ and $\mathrm{C}$ minus $\mathrm{G}$. Looking at the plot of $\mathrm{C}$ minus $\mathrm{G}$, one can also notice that solution $\mathrm{G}$ gives substantially larger errors than solution $\mathrm{C}$, leading to negative $\delta \sigma_{X}$ and $\delta \sigma_{Y}$ (and one can easily figure out that the same would be observed for $\mathrm{E}$ minus $\mathrm{G}$ ). This indicates that, although $\mathrm{G}$ significantly departs from $\mathrm{C}$ and $\mathrm{E}$ at the level of a tenth of mas, as observed in the upper plots of the $\mathrm{C}$ minus $\mathrm{G}$ figure, this 

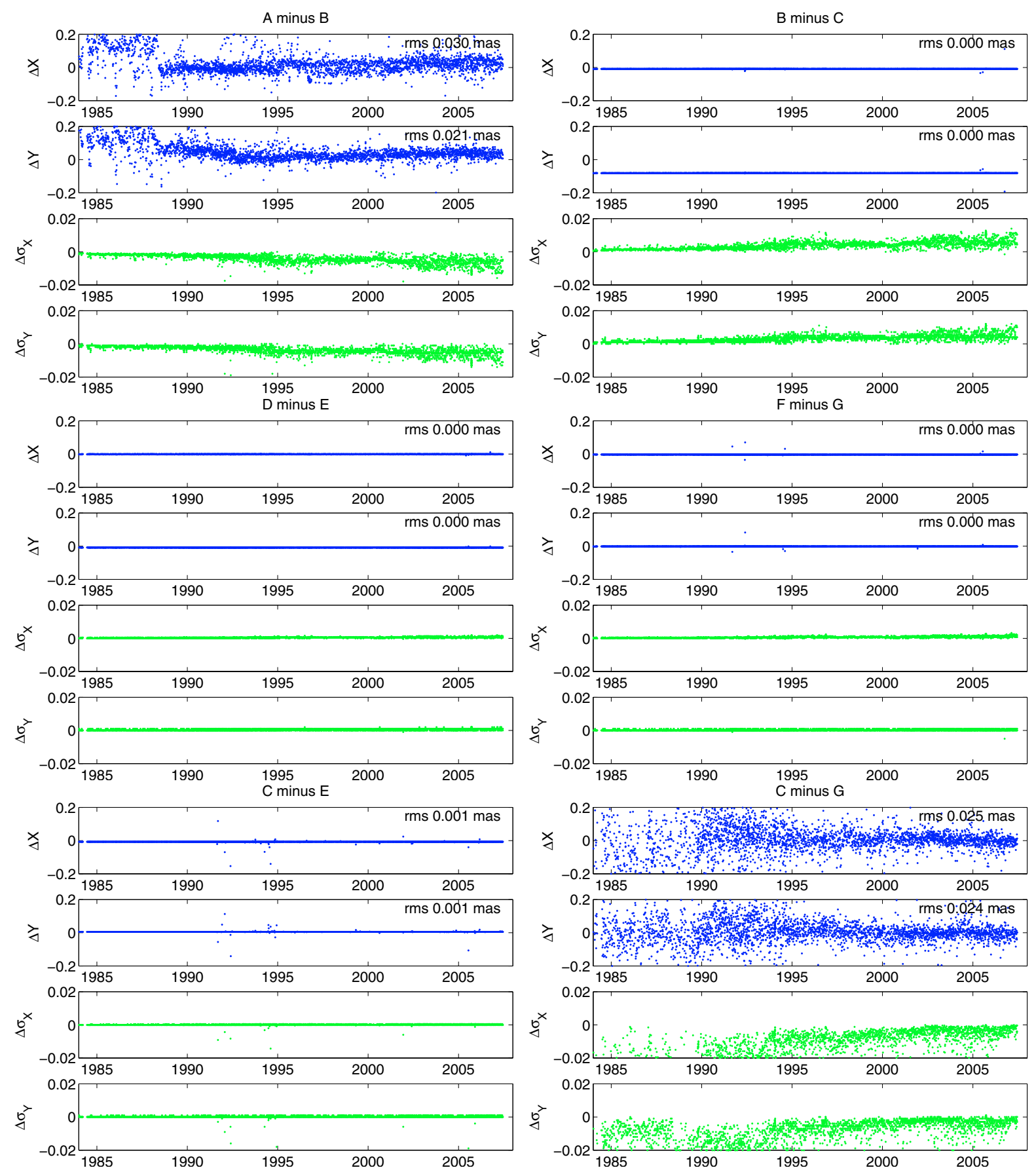

Fig. 2. Differences between nutation time series and their formal error for some solutions. Unit: mas.

departure has to be interpreted with great care in view of the associated errors.

Comparing solutions $\mathrm{B}, \mathrm{D}$, and $\mathrm{F}$ against $\mathrm{C}, \mathrm{E}$, and $\mathrm{G}$, respectively (i.e., looking at the figures titled B minus $\mathrm{C}$, D minus $\mathrm{E}$, and $\mathrm{F}$ minus $\mathrm{G}$, respectively), yields that taking the MFV stable sources instead of the ICRF defining sources for the NNR constraint does not influence the nutation offsets noticeably. Only the formal errors are noticeably affected when all the radio source coordinates are taken as global parameters in B minus $\mathrm{C}$. Moreover, the bias in $\Delta Y$ showing up on $\mathrm{B}$ minus $\mathrm{C}$ is explained by the global rotation of the reference frame around the $X$-axis (angle $A_{1}$ ) from $-22 \mu$ as to $40 \mu$ as (see Table 1). Similar reasonings can be applied to the differences between other solutions and between the formal errors associated with nutation estimates.

We then fit the amplitude of a number of nutation spectral components on the time series of nutation offsets, represented as a complex quantity

$\tilde{\eta}(t)=\delta X(t)+\mathrm{i} \delta Y(t)$

related to the complex representation of the equatorial component of the Earth's wobble $\tilde{\omega}=\omega_{x}+\mathrm{i} \omega_{y}$, expressed in a crustfixed frame of reference, by

$\frac{\mathrm{d} \tilde{\eta}(t)}{\mathrm{d} t}=-\mathrm{i} \tilde{\omega}(t) \mathrm{e}^{\mathrm{i} \Phi}$, 
Table 3. Amplitudes of the forced nutations (corrected from non linear terms).

\begin{tabular}{|c|c|c|c|c|c|c|c|c|c|c|c|c|c|c|c|c|c|c|c|c|c|}
\hline & & -18.6 & \pm & 18.6 & \pm & -9.3 & \pm & 9.3 & \pm & -6.2 & \pm & 6.2 & \pm & 1 & \pm & -1 & \pm & 0.5 & \pm & -0.5 & \pm \\
\hline \multirow{9}{*}{$\operatorname{Re}$} & $\mathrm{A}$ & 41 & 4 & -2 & 4 & 6 & 3 & 3 & 3 & -9 & 2 & 6 & 2 & -9 & 2 & 25 & 2 & 11 & 2 & -14 & 2 \\
\hline & B & 29 & 4 & 3 & 4 & 5 & 3 & 3 & 3 & -11 & 2 & 2 & 2 & -8 & 2 & 29 & 2 & 11 & 2 & -13 & 2 \\
\hline & C & 30 & 4 & 1 & 4 & 6 & 3 & 2 & 3 & -12 & 2 & 2 & 2 & -8 & 2 & 29 & 2 & 11 & 2 & -13 & 2 \\
\hline & D & 30 & 4 & 1 & 4 & 6 & 3 & 2 & 3 & -12 & 2 & 2 & 2 & -8 & 2 & 29 & 2 & 11 & 2 & -13 & 2 \\
\hline & E & 30 & 4 & 1 & 4 & 6 & 3 & 2 & 3 & -12 & 2 & 2 & 2 & -8 & 2 & 29 & 2 & 11 & 2 & -13 & 2 \\
\hline & $\mathrm{F}$ & 35 & 4 & -9 & 4 & 7 & 3 & 11 & 3 & -11 & 2 & 0 & 2 & -9 & 2 & 27 & 2 & 11 & 2 & -13 & 2 \\
\hline & G & 35 & 4 & -9 & 4 & 7 & 3 & 11 & 3 & -11 & 2 & 0 & 2 & -9 & 2 & 27 & 2 & 11 & 2 & -13 & 2 \\
\hline & Av. & 32 & 23 & -2 & 23 & 6 & 10 & 5 & 16 & -12 & 10 & 2 & 13 & -8 & 8 & 27 & 10 & 11 & 6 & -14 & 7 \\
\hline & & & 12 & & 11 & & 1 & & 8 & & 3 & & 6 & & 1 & & 4 & & 0 & & 1 \\
\hline \multirow{9}{*}{$\mathrm{Im}$} & $\bar{A}$ & -7 & 4 & 0 & 4 & 23 & 3 & -1 & 3 & 12 & 2 & 5 & 2 & 10 & 2 & -12 & 2 & 3 & 2 & 6 & 2 \\
\hline & B & -15 & 4 & -15 & 4 & 22 & 3 & -8 & 3 & 12 & 2 & 2 & 2 & 10 & 2 & -11 & 2 & 2 & 2 & 8 & 2 \\
\hline & C & -14 & 4 & -14 & 4 & 23 & 3 & -7 & 3 & 13 & 2 & 2 & 2 & 10 & 2 & -12 & 2 & 2 & 2 & 8 & 2 \\
\hline & D & -14 & 4 & -14 & 4 & 23 & 3 & -7 & 3 & 13 & 2 & 2 & 2 & 10 & 2 & -12 & 2 & 2 & 2 & 8 & 2 \\
\hline & E & -14 & 4 & -14 & 4 & 23 & 3 & -7 & 3 & 13 & 2 & 2 & 2 & 10 & 2 & -12 & 2 & 2 & 2 & 8 & 2 \\
\hline & F & -11 & 4 & -10 & 4 & 23 & 3 & -5 & 3 & 16 & 2 & 3 & 2 & 10 & 2 & -12 & 2 & 1 & 2 & 8 & 2 \\
\hline & G & -11 & 4 & -10 & 4 & 23 & 3 & -5 & 3 & 16 & 2 & 3 & 2 & 9 & 2 & -12 & 2 & 1 & 2 & 8 & 2 \\
\hline & Av. & -13 & 19 & -11 & 26 & 22 & 9 & -6 & 15 & 13 & 10 & 3 & 9 & 9 & 6 & -12 & 7 & 2 & 7 & 8 & 9 \\
\hline & & & 8 & & 15 & & 1 & & 6 & & 4 & & 2 & & 0 & & 1 & & 1 & & 2 \\
\hline
\end{tabular}

The values in bold are the estimated contribution of the celestial reference frame to the errors on nutations. Periods are in years and amplitudes in $\mu$ as.

where $\Phi$ is the sidereal rotation angle. The spectral components are circular terms of frequency $v$ and phase $\phi$, fixed by a linear combination of Delaunay variables $\left(l, l^{\prime}, F, D, \Omega\right)$, as

$\tilde{\eta}(t)=\left(A^{\operatorname{Re}}+\mathrm{i} A^{\operatorname{Im}}\right) \mathrm{e}^{\mathrm{i}(v t+\phi)}$,

wherein the amplitude $A$ is split into real and imaginary parts.

The technique employed hereafter is a weighted least-square fit, where the weights are generally computed as inversely proportional to the squared errors associated with the data. However, formal errors produced by VLBI software packages appear unrealistic when compared to the scatter (see, e.g., Herring et al. 1991, 2002). Indeed, the scatter is larger than the formal errors, yielding an extra variance not accounted for in the standard error. To remedy this discrepancy, we try to find a relationship between the formal error $\sigma$ and the scatter $\xi$ of the points having this formal error (Fig. 3). We follow the procedure already described in Herring et al. (2002) wherein the nutation offsets are binned by intervals of error (we choose intervals of $20 \mu \mathrm{as}$ ). The procedure is applied to the nutation offsets residuals obtained from a preliminary fit of the prominent nutation terms (see the next paragraph) and from which a retrograde 430.21-day term has been removed. The latter term is adjusted separately from the other, over a 2-yr sliding window, in order to account for the time-varying amplitude and phase of the free core nutation. Finally, a fit of the form $\xi^{2}=\sigma_{0}^{2}+k^{2} \sigma^{2}$ yields $\sigma_{0}=0.12$ mas and $k=1.8$ on both $X$ and $Y$ components. These values admit ignorable variations from one solution to another. They are slightly higher than in Herring et al. and most likely reflect the fact that more VLBI observations produce smaller formal errors. In the rest of the paper (and especially for the fit in the next paragraph), we scale up and translate all the VLBI data set formal errors by these values.

We then estimate prograde and retrograde amplitudes of the following forced nutation terms: 18.6-yr $(\Omega), 9.3-\mathrm{yr}(2 \Omega)$, 3.6-yr $(-2 l+2 F+\Omega)$, annual $\left(l^{\prime}\right)$, semi-annual $(2 F-2 D+2 \Omega)$, tri-annual $\left(l^{\prime}+2 F-2 D+2 \Omega\right)$, monthly $(l)$, and semi-monthly $(2 F+2 \Omega)$, jointly with a linear trend on each component and a retrograde 430.21-day term.

Once the amplitudes are obtained, we remove the contribution coming from the non linear terms, accounting for the effects
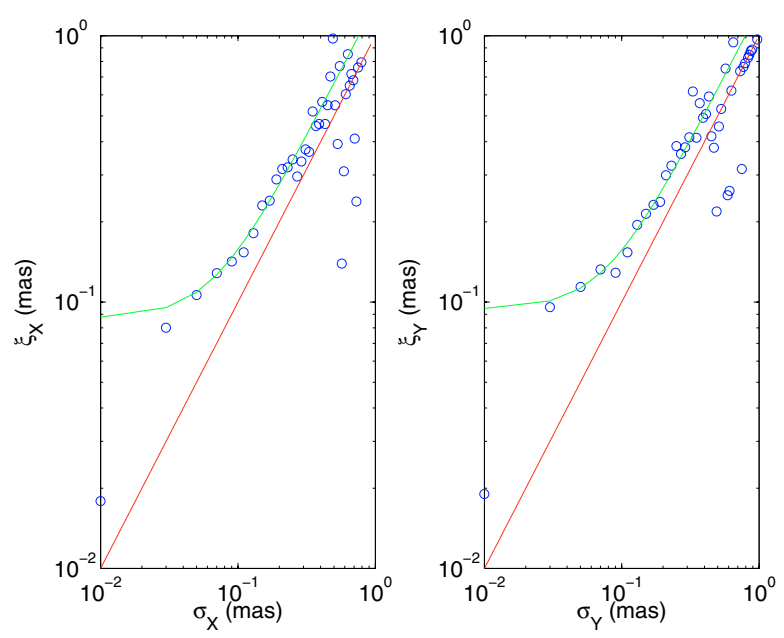

Fig. 3. Scatter of nutation offsets versus their formal error (blue circles). The green, solid line is the fit of $\xi^{2}=\sigma_{0}^{2}+k^{2} \sigma^{2}$.

of the external torque on the tidal redistribution. This contribution was not completely taken into account in MHB, since only the effects of the tesseral potential on sectorial and zonal tides were computed. Their reciprocal effects (zonal and sectorial potentials on tesseral tides) have been established in Lambert \& Mathews (2006). Because of an almost perfect cancelation of the reciprocal effects, the net effect amounts to $\pm 7 \mu$ as on the imaginary parts of the $\pm 18.6-y$ r nutations, respectively (compared to $34 \mu$ as and $-4 \mu$ as of MHB). To free our nutation residuals from these non linear effects, the difference ( $27 \mu$ as and $3 \mu$ as on the imaginary parts of \pm 18 .6-yr nutations, respectively) therefore has to be added to the previously estimated amplitudes.

Table 3 and Fig. 4 gather the obtained amplitudes of the forced nutation terms (against MHB) adjusted in solutions A to $\mathrm{G}$ (in blue line). It appears that the largest offsets to MHB are on the prograde annual and on the retrograde 18.6-yr nutations. The thick, red line represents an average of the solutions (line tagged as Av. in Table 3), the associated red error bars represent the cumulated error due to (i) the least-square fit (estimated as the square root of the sum of the squared formal errors) and (ii) to 

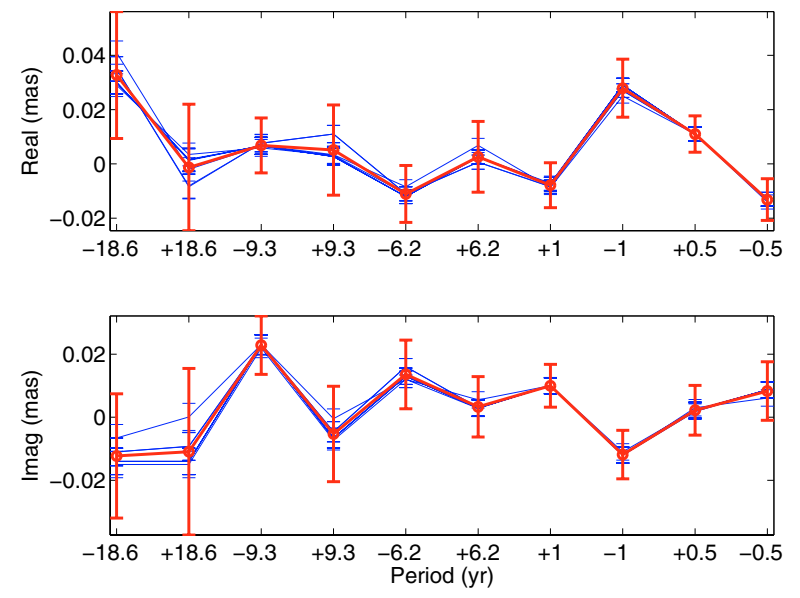

Fig. 4. Amplitude of the VLBI residuals for prominent nutation periods against MHB from solutions A to G. The thick, red line is the averaged amplitude.

the unperfect realization of the celestial frame. This contribution is obtained as the difference between the maximum and minimum amplitudes for each frequency and is reported in Table 3 (values in bold). As already mentioned earlier, the influence of the celestial frame for annual and smaller periods is weaker than for longer periods.

The method of retrieving the outer and inner core resonant frequencies is based on the transfer function that expresses the ratio between rigid and non rigid nutation amplitudes (resp. $\tilde{\eta}_{R}$ and $\left.\tilde{\eta}_{N R}\right)$ in the frequency domain:

$\tilde{\eta}_{N R}(v)=T(v) \tilde{\eta}_{R}(v)$,

where $v$ is the forcing frequency expressed in the Earth-fixed frame of reference. The formula of the transfer function corresponds to Eq. (42) of the MHB paper

$T(v)=\frac{e_{R}-v}{e_{R}+1} N_{0}\left[1+(1+v)\left(Q_{0}+\sum_{\alpha=1}^{4} \frac{Q_{\alpha}}{v-v_{\alpha}}\right)\right]$,

with nominal values of the parameters taken in Table 6 of the same paper ( $e_{R}$ is the "rigid" ellipticity assumed in the construction of the rigid nutation theory). The $v_{\alpha}$ are the eigenfrequencies of the three-layer Earth system (Chandler and inner core wobbles acting at long periods in the Earth-fixed frame of reference, and the RFCN and FICN in the retrograde diurnal band). For a given forcing frequency $v$, the observed correction to the MHB transfer function is obtained by dividing the fitted amplitude by the rigid counterpart taken from Souchay et al. (1999), consistent with what was done in the MHB work. Then the observation equations are obtained by computing the partial derivatives of $T(v)$ with respect to $v_{\mathrm{RFCN}}$ and $v_{\mathrm{FICN}}$, all the other parameters in the transfer function being fixed to their MHB value.

The obtained values of the resonant RFCN and FICN periods $P$ and quality factors $Q$ are reported in Table 4 and in Fig. 5 wherein the uncertainties represent the formal error of the least-square fit. The influence of this analysis strategy can be seen by comparing $P$ and $Q$ from one solution to the next. One can see that the RFCN period goes from -430.30 to -430.32 days, an interval smaller than the least-square standard error, which amounts to 0.08 day. The quality factor is stable within less than 200. The FICN period stays between 1042 and 1113 days (with a formal error around 120 days). Its quality factor is between 885 and 974 with error bars of 200 .
Table 4. Resonant period $P$ (in days) and quality factor $Q$ of the RFCN and of the FICN estimated from various VLBI solutions A to G.

\begin{tabular}{rrrrrrrrr}
\hline \hline & & RFCN & & \multicolumn{4}{c}{ FICN } \\
& $P$ & \pm & $Q$ & \pm & $P$ & \pm & $Q$ & \pm \\
\hline $\mathrm{A}$ & -430.32 & 0.08 & 18989 & 352 & 1113 & 134 & 885 & 197 \\
$\mathrm{~B}$ & -430.30 & 0.08 & 18823 & 343 & 1042 & 110 & 958 & 207 \\
$\mathrm{C}$ & -430.30 & 0.08 & 18821 & 346 & 1047 & 117 & 972 & 214 \\
$\mathrm{D}$ & -430.30 & 0.08 & 18822 & 346 & 1047 & 116 & 971 & 214 \\
$\mathrm{E}$ & -430.30 & 0.08 & 18821 & 346 & 1047 & 117 & 974 & 215 \\
$\mathrm{~F}$ & -430.31 & 0.08 & 18875 & 353 & 1076 & 138 & 951 & 218 \\
$\mathrm{G}$ & -430.31 & 0.08 & 18876 & 353 & 1076 & 138 & 954 & 219 \\
\hline
\end{tabular}
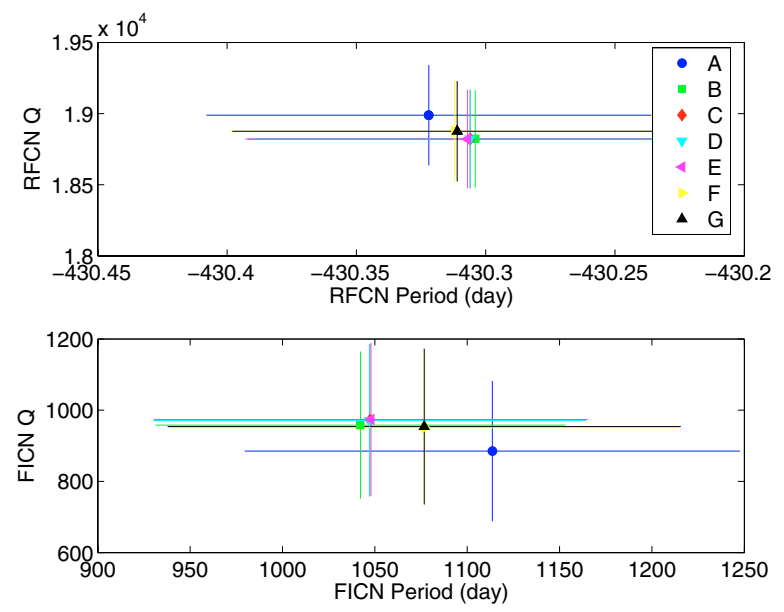

Fig. 5. Resonant period and quality factor of the RFCN and of the FICN estimated from various VLBI solutions A to $\mathrm{G}$.

\section{Conclusion}

Our work points out the order of magnitude of the error coming from the positional instability of the radio sources and the handling of the celestial frame in VLBI-derived nutation analyses for use in geophysics. This error can produce an additional error in the estimates of nutation spectral components of $15 \mu$ as for the 18.6-yr term that decreases for shorter periods. In terms of resonant frequencies of the outer and inner cores, this means an uncertainty of a few tenths of a day on the RFCN period and of 200 on its quality factor, and an uncertainty of less than 100 days on the FICN period and of 100 on its quality factor. The values yielded for the resonant frequencies are nevertheless close to the MHB values within the error bars.

Although not within the scope of this paper, finding the best strategy for accounting for radio source instabilities in geodetic VLBI analysis is a challenging question for the near future (including possibilities of directly correcting the delay from radio source structure delay). Solutions A to G were processed with analysis strategies that are currently in use through the VLBI community. It is expected that some of these analysis strategies bring substantial errors to the Earth orientation parameters due to incorrect realizations of reference frames. In that sense, some operational solutions proposed within the VLBI community will be more reliable for geophysical investigations since they bring Earth orientation parameters with a best internal accuracy. Finally, the reader has to keep in mind that the number of analysis strategies shown in this paper is not exhaustive: one should also consider many other potential sources of error (e.g., troposphere gradients, network geometry).

Acknowledgements. This study could not have been carried out without the almost 30-yr long, great work of the geodetic VLBI community, now concretely 
gathered around the International VLBI Service for Geodesy and Astrometry (IVS) that coordinates the observations, correlates and stores the data, as well as all the participating network stations over the world. We also thank an anonymous referee for the interesting and constructive remarks and comments on this work.

\section{References}

Capitaine, N., Chapront, J., Lambert, S. B., \& Wallace, P. T. 2003, A\&A, 400, 1145

Feissel-Vernier, M. 2003, A\&A, 422, 1105

Feissel-Vernier, M., Ma, C., Gontier, A.-M., \& Barache, C. 2006, A\&A, 452, 1107

Fey, A. L., Ma, C., Arias, E. F., et al. 2004, AJ, 127, 3587

Gegout, P., Hinderer, J., Legros, H., et al. 1998, PEPI, 106, 337

Gontier, A.-M., Le Bail, K., Feissel, M., \& Eubanks, T. M. 2001, A\&A, 375, 661

Herring, T. A., Buffett, B. A., Mathews, P. M., \& Shapiro, I. I. 1991, J. Geophys. Res., 96, 8259
Herring, T. A., Mathews, P. M., \& Buffett, B. A. 2002, J. Geophys. Res., 107(B4), 10.1029/2001JB000165

IERS 1996, International Earth Rotation Service Annual Report 1995 (Observatoire de Paris), II-19

Lambert, S. B., \& Mathews, P. M. 2006, A\&A, 453, 363

Lambert, S. B., \& Dehant, V. 2007, A\&A, 469, 777

Ma, C., Arias, E. F., Eubanks, T. M., et al. 1998, AJ, 116, 516

MacMillan, D. S., \& Cohen, S. 2004, in International VLBI Service for Geodesy and Astrometry (IVS) 2004 General Meeting Proceedings, ed. N. R. Vandenberg, \& K. D. Baver, NASA/CP-2004-212255, 491

Mathews, P. M., Herring, T. A., \& Buffett, B. A. 2002, J. Geophys. Res., 107(B4), 10.1029/2001JB000390

Niell, A. 1996, J. Geophys. Res., 101(B2), 3227

Nothnagel, A. 2005, in Proceedings of the 17th Working Meeting on European VLBI for Geodesy and Astrometry, ed. M. Vennebusch, \& A. Nothnagel, 118 Schlueter, W., Himwich, E., Nothnagel, A., et al. 2002, Adv. Space Res., 30, 145 Souchay, J., Loysel, B., Kinoshita, H., \& Folgueira, M. 1999, A\&AS, 135, 111 Vondrák, J., Weber, R., \& Ron, C. 2005, A\&A, 444, 297 